Meta

Journal des traducteurs

Translators' Journal

\title{
Point de vue en langue spécialisée
}

\section{Anne Condamines et Josette Rebeyrolle}

Volume 42, numéro 1, mars 1997

Lexicologie et terminologie

URI : https://id.erudit.org/iderudit/002359ar

DOI : https://doi.org/10.7202/002359ar

Aller au sommaire du numéro

Éditeur(s)

Les Presses de l'Université de Montréal

ISSN

0026-0452 (imprimé)

1492-1421 (numérique)

Découvrir la revue

Citer cet article

Condamines, A. \& Rebeyrolle, J. (1997). Point de vue en langue spécialisée. Meta, 42(1), 174-184. https://doi.org/10.7202/002359ar

\section{Résumé de l'article}

Cet article aborde la question de la polysémie dans les langues spécialisées. La notion du point de vue permet de faire un premier point sur des différences de fonctionnement en langue générale et en langue spécialisée. En conclusion, il rend compte d'une méthode de mise en évidence de cas de polyacceptions, et de l'existence de points de vue collectifs. 


\title{
POINT DE VUE EN LANGUE SPÉCIALISÉE
}

\author{
ANNE CONDAMINES* ET JOSETTE REBEYROLLE** \\ *Centre National de la Recherche Scientifique et \\ **Université Toulouse le Mirail. Toulouse, France
}

Résumé

Cet article aborde la question de la polysémie dans les langues spécialisées. La notion du point de vue permet de faire un premier point sur des différences de fonctionnement en langue générale et en langue spécialisée. En conclusion, il rend compte d'une méthode de mise en évidence de cas de polyacceptions, et de l'existence de points de vue collectifs.

\begin{abstract}
This article looks at polysemy in specialized languages using the concept of point of view to examine differences between general language and specialized language. It concludes with a method for highlighting examples of multiple meanings and collective points of view.
\end{abstract}

\section{INTRODUCTION}

Le terme de point de vue est très utilisé dans les travaux actuels en intelligence artificielle (IA) où il s'emploie dans un sens assez proche de celui de la langue commune. Sur beaucoup de questions, des rapprochements ont été faits entre intelligence artificielle et terminologie, qui partagent dans leur objet d'étude le fait de travailler sur des domaines spécialisés. Dans cet article, laissant de côté les problèmes spécifiques de l'IA, nous étudions ce que peut recouvrir la notion de point de vue dans les langues spécialisées. Nous examinons d'abord comment cette notion apparaît dans les travaux de linguistique générale, en particulier en sémantique et dans les travaux sur l'énonciation. Dans un second paragraphe, nous examinons ce que devient cette même notion en terminologie, lexique d'une langue spécialisée dont la principale caractéristique est d'être parlée par des locuteurs identifiés (ou, du moins, identifiables). Après une rapide réflexion sur la question langue spécialisée ou discours spécialisé, nous abordons les notions de polysémie et de polyacception. Nous identifions deux types de polyacceptions dans les langues spécialisées, l'une reliée au point de vue du locuteur de la langue spécialisée (caractérisé par une connaissance spécifique), l'autre au point de vue d'un locuteur particulier à l'intérieur d'un domaine particulier. Le dernier paragraphe présente une expérimentation qui a permis d'une part de mettre en œuvre une méthodologie de repérage de polyacceptions, et d'autre part de démontrer l'existence d'un point de vue en lien avec une connaissance particulière (le point de vue privilégié par le locuteur de ce domaine particulier).

\section{POINT DE VUE EN LINGUISTIQUE}

En sémantique, deux grands types d'approches théoriques et descriptives caractérisent la plurivocité des expressions lexicales: l'homonymie et la polysémie. On parle de polysémie lorsque, à un signifiant unique correspondent plusieurs sens perçus comme étant reliés entre eux, et d'homonymie s'il n'y a pas de liens entre les sens identifiés. Ces deux types d'approches posent le problème de la nature des relations que les différents sens entretiennent (ou pas). La question de la définition de ces relations, qui enregistre une diversité de réponses dans les études lexicales, conditionne la distinction entre homonymie 
et polysémie. Ainsi, la réponse peut être donnée en termes d'étymologie, si deux mots possèdent un étymon commun, ils sont dits polysémiques (p. ex. Martinet 1974; Lyons 1990). Elle peut s'appuyer sur des critères morphologiques (dérivés): si les différents sens correspondent à des constructions différentes et donnent lieu à des dérivés spécifiques, alors ils sont attribués à des termes homonymes (p. ex. Guilbert 1973), ou sur des critères sémantiques paradigmatiques (des polysèmes ont des synonymes identiques et des antonymes identiques) (p. ex. Baylon et Fabre 1978; Lerat 1983). La réponse peut également s'appuyer sur la notion de sèmes: la polysémie suppose donc une cohérence sémique qui manque aux homonymes (p. ex. Picoche 1984; Lyons 1990). Elle peut être expliquée par des principes sémantico-logiques de dérivation qui permettent de définir les liens de sens qui unissent les divers signifiés d'une même forme polysémique : pour rendre compte de la polysémie des unités lexicales, on considère les modifications qui peuvent s'opérer sur le sème central d'un mot, par des glissements de sens du type métaphore, métonymie, spécialisation, extension, restriction, etc. (p. ex. Martin 1972; Kerbrat-Orecchioni 1977).

Cette distinction (polysémie/homonymie) est essentielle en linguistique, notamment dans la manière de concevoir les entrées du dictionnaire. Ces variantes de conception se traduisent souvent par des écarts sensibles dans la répartition des polysèmes et des homonymes d'un dictionnaire à l'autre : là où le lexicographe reconnaît des homonymes, on aura autant d'articles séparés; tandis que là où il ne trouve qu'un mot unique polysémique, il n'y aura qu'une seule entrée.

Si un mot peut avoir plusieurs sens en langue, il faut se poser, en discours, la question de savoir comment l'interlocuteur peut se rendre compte de la signification pour laquelle a opté le locuteur. La structure syntagmatique précise alors le choix du sens dans une situation linguistique donnée: «Chaque emploi d'une unité signifiante vaut en fonction du choix qu'elle représente parmi celles qui auraient pu figurer dans le même environnement» (Martinet 1974: 44). La polysémie suppose donc qu'il y a plusieurs valeurs de sens possibles parmi lesquelles l'une est choisie, actualisée dans le discours, en fonction de ce que l'on peut considérer comme un point de vue particulier. Ainsi, comme le remarque Saussure, selon la manière dont on le considère, le mot $n u$ sera un son, l'expression d'une idée, le correspondant du latin nudum, etc. : «Bien que l'objet précède le point de vue, on dirait que c'est le point de vue qui crée l'objet» (Saussure 1983: 23).

Dans d'autres types de travaux, les travaux sur l'énonciation «le point de vue, qui correspond à l'énonciateur de Ducrot, désigne le personnage dont le récit exprime les opinions, sans que ce personnage soit nécessairement confondu avec le narrateur» (Moeschler et Reboul 1994: 426). Même s'il n'est pas forcément identifiable en tant que personne en chair et en os, l'énonciateur, qui est assimilé ici au point de vue, a des opinions et une compétence d'observation qui «impliquent un savoir limité» (Lintvelt 1981:96).

Les travaux concernant l'énonciation se rapportent à une théorie du discours et non de la langue. Or, nous pensons que les langues spécialisées sont aussi des discours spécialisés ( $c f .2 .2$.).

\section{POINT DE VUE EN TERMINOLOGIE}

S'il existe une parenté évidente entre langue et langues spécialisées, un certain nombre d'éléments qui tiennent en grande partie à la spécificité du contexte d'utilisation de ces langues spécialisées doivent être clairement explicités. La présentation de cette spécificité nous permettra de situer comment nous abordons la notion de point de vue en langues spécialisées.

\subsection{SPÉCIFICITÉ DES LANGUES SPÉCIALISÉES : UN LOCUTEUR IDENTIFIÉ}

Le fait que les langues spécialisées soient avant tout «de la langue» est désormais généralement reconnu1. Toutefois, la difficulté même que nous avons eue à formuler cette 
relation langue spécialisée/langue manifeste qu'elle n'est pas si aisée à établir. Si, comme le pensent plusieurs auteurs dont nous partageons l'avis, une langue spécialisée ne constitue pas une langue à part entière parce qu'elle ne constitue en aucune façon un système autonome, comment caractériser alors sa spécificité ?

Il semble bien que l'élément le plus important de cette spécificité tienne à ce qu'une langue spécialisée est parlée par des locuteurs identifiables à leur rôle social par des éléments reliés à leur compétence. Toutes les études sur les langues spécialisées portent sur des textes (entendus au sens large, donc y compris des transcriptions d'entretiens avec des experts) qui ont été réellement produits par des locuteurs évoluant dans le domaine dans lequel s'inscrit la langue spécialisée. Le travail ne s'effectue pas, comme pour les études de langue générale, sur seulement quelques phrases produites par le linguiste faisant alors office de locuteur idéal. Même dans le cas où, en langue spécialisée, on veut tester l'acceptabilité d'une phrase non encore produite, c'est à un expert du domaine que l'on s'adresse.

Remarque: on peut se demander alors si le travail sur corpus ne relève pas toujours d'une linguistique du discours. En effet, on connaît toujours l'origine d'un corpus, c'està-dire le contexte socioculturel dans lequel il a été écrit, de quel genre il relève... «La situation de production n'est pas neutre, et ne peut être définie abstraitement. Elle prend toujours place dans une pratique sociale qui définit le discours dont relève le texte, et le genre qui le structure» (Rastier 1995: 13).

Etant donné que le travail d'analyse de langues spécialisées s'effectue toujours à partir de corpus, on peut penser qu'en réalité, ce travail relève toujours d'une linguistique du discours et qu'il vaudrait mieux parler de discours spécialisés que de langues spécialisées.

\subsection{LANGUE SPÉCIALISÉE OU DISCOURS SPÉCIALISÉ ?}

Question redoutable, au centre de toute la problématique des langues spécialisées et qui mériterait un long développement qui ne sera pas fait ici. Nous nous contenterons de l'aborder à travers la problématique du point de vue.

D'une certaine façon, ainsi que nous l'avons vu en 2.1 , notre incompétence dans les domaines étudiés nous oblige à étudier la langue à travers ses actualisations, telle qu'elle se manifeste dans les corpus. Nous n'avons accès qu'à du discours. Par ailleurs, le système auquel obéissent ces manifestations n'est pas autonome, il dépend toujours d'une langue préexistante. Ces deux arguments amènent à considérer les langues spécialisées comme une actualisation de la langue, c'est-à-dire un discours. Dans ce cas, on retrouve la notion de point de vue que l'on a évoquée en 1, celle qui existe lorsque l'actualisation de la langue passe par des choix qui manifestent des points de vue. Mais ici, ce point de vue est associé à un groupe de locuteurs qui partagent une même compétence, celle du domaine dans lequel est utilisée la langue spécialisée.

Or, s'il y a compétence cognitive commune, elle doit se manifester par une compétence linguistique commune. Compétence et non performance. Cette compétence, c'est à la fois celle de la langue générale et celle du lexique spécialisé, deux éléments qui constituent un système (un sous-système) que l'on va pouvoir continuer à appeler langue spécialisée.

Ainsi, dans une première abstraction, la langue générale s'actualise dans un discours (un discours spécialisé) qui, parce qu'il est utilisé dans un contexte cognitif déterminé, va donner naissance, dans une deuxième abstraction, à un système linguistique adapté appelé langue spécialisée. Il y a un premier type de point de vue dans l'actualisation de la langue générale en discours spécialisé, un point de vue qui intéresse au plus haut point le terminologue car il est lié à une conception particulière, celle qui est en cours dans le domaine à l'étude. Il y a un second type de point de vue qui concerne l'actualisation de la langue spécialisée en discours, en fonction d'un locuteur particulier. Ce point de vue-là ne 
manifeste pas une connaissance mais simplement un choix linguistique qui relève de l'argumentation, tout comme dans le passage de la langue générale au discours d'un locuteur particulier.

Ainsi que nous l'avons vu en 1, le point de vue est toujours en lien avec un choix; celui-ci peut être individuel, ne concerner qu'un locuteur ou bien être collectif et concerner un groupe de locuteurs. C'est ce second type de point de vue qui sera plus intéressant pour la terminologie.

Quant à la question langues spécialisées ou discours spécialisés, nous répondrons provisoirement que l'objet que nous étudions possède à la fois certaines caractéristiques des langues et certaines caractéristiques des discours.

\subsection{POLYSÉMIE DANS LES LANGUES SPÉCIALISÉES ?}

Si, comme l'écrit Lerat (1995: 20), «la langue spécialisée est une langue naturelle considérée en tant que vecteur de connaissances spécialisées», on voit mal pourquoi ces langues échapperaient complètement au phénomène de la polysémie. Pourtant, nombre d'auteurs posent fermement comme postulat qu'il n'y a pas de polysémie en langue spécialisée: «L'unité terminologique est, par essence, monosémique alors que le mot en tant qu'unité linguistique est voué à la polysémie» (Guilbert 1981 in Lerat 1995: 93). Cette citation laisse entendre que le terme ne serait pas une unité linguistique; or, même si on peut reconnaître que le terme possède un fonctionnement particulier, puisqu'il entretient une relation privilégiée avec la connaissance d'un domaine, on voit mal comment on pourrait lui ôter son statut de signe linguistique. Le simple fait de passer de la langue générale à une langue spécialisée ne peut pas faire perdre au terme son statut de signe linguistique.

Certains auteurs, beaucoup plus rares, évoquent la possibilité de l'existence de la polysémie dans les langues spécialisées:

«Le caractère monosémique a souvent été attribué aux termes, pour les opposer au fonctionnement polysémique qui marque, au contraire, d'une façon générale, le lexique des langues naturelles; mais cette affirmation appelle un certain nombre de précisions, voire de restrictions» (Mortureux 1995).

Notons toutefois qu'il nous a été très difficile de trouver des travaux qui traitent explicitement de la polysémie en terminologie. Certains évoquent des effets de sens, que l'on pourrait considérer comme la manifestation d'un phénomène polysémique :

«Par nature, un terme est ambivalent. Il joue d'une part un rôle de lexie et admet à ce titre une définition lexicologique qui, en discours, $n$ 'interdit pas les effets de sens...» (Gentilhomme 1994 : 557).

Si l'existence de polysémie en langue est corrélée avec celle de point de vue, et si une langue spécialisée est aussi une langue, alors, l'existence de polysémie en langue spécialisée est aussi à corréler avec l'existence du point de vue.

Dans quelques travaux transparaît la notion de point de vue, comme dans l'extrait suivant: Beacco et Moirand constatent que «dans un domaine précis, il n'existe pas un discours de spécialité, mais des discours qui s'entrecroisent et varient en fonction des positions des locuteurs, des relations entre interlocuteurs, des situations de communication rencontrées» (Beacco et Moirand 1995: 34).

On voit tout de suite que la réflexion porte ici sur l'existence d'un point de vue relié à un locuteur. De même, Gentilhomme, dans l'extrait précédent, parlait d'effets de sens en discours. Ces citations montrent clairement que ces auteurs travaillent sur des discours et qu'ils étudient donc des discours spécialisés plutôt que des langues spécialisées. 


\subsection{POLYSÉMIE ET ANALYSE DE CORPUS SPÉCIALISÉS}

Tout comme pour une langue générale, analyser un corpus pour y examiner le phénomène de la polysémie revient à repérer des termes et à procéder à un classement des contextes dans lesquels ils apparaissent afin d'identifier si ces contextes peuvent être considérés comme sémantiquement homogènes ou non. L'exploration contextuelle que nous proposons est assez comparable à celle mise en ouvre par l'équipe de J.-L. Descamps (Descamps et coll. 1992). Cette exploration vise à essayer de dégager un fonctionnement régulier à travers des manifestations linguistiques variables. Il s'agit d'essayer de cerner le sens d'un mot en s'appuyant sur les connaissances linguistiques que l'on a sur le contextc.

En cas d'homogénéité des contextes d'apparition d'un terme, il n'y aura pas de polysémie; dans le cas contraire, il y aura homonymie ou polysémie. La polysémie est déduite comme étant une des caractéristiques de la langue dont le discours est une actualisation. Ce qui apparaît dans le texte, c'est le résultat du choix qui a été fait, la manifestation du point de vue adopté. Si l'on tient compte de ce que nous écrivons en 2.2 , toute la difficulté, dans les corpus spécialisés, sera de repérer si le point de vue est spécifique à un locuteur particulier ou à un ensemble de locuteurs partageant les mêmes compétences.

L'analyse de corpus pour l'étude d'une éventuelle polysémie permet de repérer, pour chaque mot ou terme, si l'on a affaire à une ou plusieurs acceptions. Mais qu'appelle-t-on acception?

«Acception : on dit d'un mot qu'il a plusieurs acceptions quand il a plusieurs sens différents selon les contextes. Un mot qui a plusieurs acceptions est polysémique; celui qui n'en a qu'une est monosémique» (Dictionnaire Larousse de Linguistique et des Sciences du Langage).

Cette définition pose clairement que la notion d'acception est liée à celle de contexte. Mais, d'une part, elle n'évoque pas la difficulté qu'il peut y avoir à évaluer des contextes pour décider combien de types on peut en identifier pour chaque mot; d'autre part, elle assimile un peu trop hâtivement le fait que des acceptions multiples manifestent des différences de sens et donc, de la polysémie. Il serait plus juste de dire, à la suite de la sémantique générative, que dans les différentes acceptions, ce ne sont pas les mêmes sèmes qui sont actualisés. Nous appelons polyacception le fait que plusieurs classes sémantiques de contextes puissent être identifiées pour un terme. Le prochain paragraphe nous permettra de mieux préciser notre démarche sur cette question.

Nous défendons la position suivante : étudier le fonctionnement des termes dans des corpus revient, pour l'essentiel, à étudier les contextes dans lesquels ils apparaissent et à les classer. Ce à quoi peut conduire cette étude, c'est à identifier qu'il peut exister une polyacception pour certains termes. Cette polyacception est la manifestation de points de vue différents, qui peuvent être associés soit à un choix individuel soit à un choix collectif à relier à des compétences socioprofessionnelles communes à un groupe identifiable de locuteurs.

\section{POINTS DE VUE EN LANGUE SPÉCIALISÉE}

À partir de l'étude d'un exemple, ce paragraphe va nous permettre de montrer comment on peut identifier qu'il y a polyacception pour un terme et comment cette polyacception peut être associée à des points de vue différents.

\subsection{REPÉRAGE DE LA POLYACCEPTION}

Comment repérer qu'il existe plusieurs acceptions pour un même terme?

En travaillant sur les contextes d'un même signifiant (c'est-à-dire une forme linguistique dont on ne sait rien a priori), plusieurs types de fonctionnement peuvent être distingués : 
a. Les contextes dans lesquels apparaît le terme prouvent que le terme appartient à deux catégories grammaticales; c'est un cas facile à identifier; il y a seulement homonymie. Par exemple, dans la langue générale, porte est à la fois nom et verbe.

b. Dans tous les contextes, le terme appartient à une même catégorie grammaticale, mais ces contextes constituent deux ou plusieurs classes syntaxico-sémantiques, clairement distinctes, sans aucune parenté. Dans ce cas, il s'agit d'une homonymie.

c. Les contextes d'apparition constituent une classe sémantiquement homogène qui permet d'identifier un seul signifié et de donner une seule définition au terme. Le terme n'admet qu'une acception; il n'est pas polysémique.

Remarque : inversement, le repérage de contextes d'apparition sémantiquement homogènes entourant des signifiants différents permet de dire que les termes correspondant à ces signifiants sont synonymes (ou au moins équivalents si l'on tient compte de la provenance des documents).

d. Les contextes d'apparition constituent deux ou plusieurs classes sémantiques qui entretiennent des parentés. L'existence d'une parenté des contextes permet d'avancer que, dans ces différents usages, les différentes acceptions du terme manifestent des traits sémantiques identiques; le terme est donc polysémique.

Remarque: il faut bien évidemment exclure de l'examen les contextes sémantiquement peu chargés qui n'apportent que des informations secondaires sur le concept à traiter et qui, d'une certaine façon, neutralisent la polysémie ou l'homonymie existante. Soit, par exemple, le mot feuille et ces deux sens nettement mis en évidence dans les deux contextes suivants :

Les arbres commencent à perdre leurs feuilles

Max écrit sur des feuilles quadrillées.

Un contexte comme «Max ramasse une feuille tombée à terre» n'est pas suffisant pour repérer quel sens est actualisé. Il est trop général; en effet, n'importe quel objet suffisamment léger a la propriété de tomber et de pouvoir être ramassé.

Cette remarque est valable à la fois pour le repérage de la polysémie et de l'homonymie (d'ailleurs, les deux acceptions de feuille manifestent-elles une homonymie ou une polysémie?).

La définition de classes de contextes, que nous posons comme un des éléments clés dans le travail d'exploration des textes, n'est pas facile à faire; elle fait appel à la faculté de généralisation du linguiste qui doit déterminer des convergences de fonctionnements syntaxico-sémantiques à travers des diversités de manifestations lexico-syntaxiques. Cette tâche peut être assistée par des outils, en particulier des outils d'analyse de contenu, qui font intervenir des calculs statistiques très complexes. Il reste que l'interprétation des résultats, le fait de donner un sens à ces résultats statistiques, incombe entièrement au linguiste.

\subsection{EXPÉRIMENTATION}

Ce paragraphe va nous permettre de présenter une méthode de mise en évidence de polyacception puis de montrer comment cette polyacception peut être mise en relation avec le point de vue d'un groupe professionnel identifié.

\subsubsection{Contexte de l'étude}

L'étude des points de vue que nous présentons a été réalisée au Centre National d'Études Spatiales (CNES).

Deux corpus, de deux divisions différentes : la division «Observation de la Terre» et la division «Mathématiques Spatiales», ont été analysés à l'aide d'outils. Nous nous sommes 
particulièrement attachées à repérer des cas de polyacceptions et à identifier s'ils étaient à rattacher à l'existence d'un point de vue individuel (non pertinent terminologiquement) ou à l'existence d'un point de vue collectif (pertinent terminologiquement). Le compte rendu que nous faisons porte sur l'étude du terme satellite. Nous montrons d'abord quelles acceptions peuvent être repérées, puis nous étudions si ces acceptions peuvent correspondre à des points de vue collectifs.

\subsubsection{Mise en évidence d'un cas de polyacception}

Dans le cadre de cette étude, nous avons utilisé le logiciel d'analyse de données textuelles ALCESTE (Analyse Lexicale par Contexte d'un Ensemble de Segments de Texte) $)^{2}$, mis au point par Max Reinert. L'objectif principal de ce logiciel est de classer les énoncés d'un fichier de textes en fonction des ressemblances et dissemblances des mots de ces énoncés.

Alceste met en œuvre une analyse statistique qui permet de réaliser un découpage du corpus de textes en différentes parties thématiquement homogènes, en autant de classes qui constituent des sous-corpus dont il est possible d'étudier la composition. Pour notre expérimentation, nous avons décidé de considérer comme corpus l'ensemble des contextes (définis arbitrairement par un nombre de chaînes de caractères) d'apparition d'un terme présumé polysémique. Les critères que nous avons retenus pour sélectionner des candidats à la polyacception sont les suivants: ces termes apparaissent dans chacune des classes avec une fréquence supérieure à une centaine d'occurrences, d'une part, et, d'autre part, le Chi2 de ces items est significatif dans au moins une des classes (chaque classe étant représentée par des mots dont le Chi2 est significatif). Le Chi2 est un indice de cohérence de classe calculé en fonction de la présence ou de l'absence des mots significatifs du corpus qui est utilisé pour estimer le lien d'appartenance d'un mot à une classe. Ainsi, au terme de l'analyse sur Alceste, nous avons retenu les termes suivants: orbite, navigation, analyse de mission, spécification, satellite.

Nous avons ensuite constitué autant de corpus que de termes candidats à la polyacception. Notre hypothèse était que les classes définies par Alceste pour chaque corpus correspondaient à autant de classes dans lesquelles le terme apparaissait avec une acception différente (pour plus de précisions sur la méthodologic de ce repérage de la polyacception assistée par outils, $c f$. Rebeyrolle 1995).

Nous ne donnons ici que les résultats concernant l'un des termes candidats à la polyacception: satellite. À partir des classes réalisées par Alceste, nous avons pu définir six patrons syntaxico-sémantiques manifestant la validité de l'organisation en classes proposée par Alceste.

Voici les six patrons syntaxico-sémantiques identifiés, caractéristiques des six types d'acceptions. Chaque patron est suivi d'exemples extraits du corpus étudié :

\section{V de type projeter dans l'espace + dét $+\mathrm{N} 1$}

(1) lancer des satellites en orbite

\section{Vsupport de type faire + dét + (déverbal) DVB de type projeter dans l'espace + de + dét + N1}

(2) effectuer le lancement du satellite

(3) effectuer le tir de SPOT4

\section{V de situation dans l'espace + dét $+\mathbf{N 1}$}

(4) positionner le satellite

(4) localiser le satellite 


\section{Vsupport de type calculer + dét + DVB + de + dét + N1}
(6) déterminer la position du satellite
(7) calculer la position du satellite
(8) prédire la position du satellite
(9) calculer, délivrer une estimation de [la] position du satellite

3. V de type placer + dét $+\mathbf{N} \mathbf{2}+$ sur + dét $+\mathbf{N 1}$

(10) placer un système sur le satellite

(11) placer un senseur stellaire sur le satellite

(11 bis) poser des fixations sur le satellite

\section{Vsupport de type faire + dét + DVB placer + de + N2 + sur + dét + N1}

(12) réaliser l'embarquement du prototype sur le satellite SPOT4

(13) faire l'intégration des instruments sur les satellites porteurs

(14) faire l'intégration des essais électriques, essais mécaniques sur le satellite

(15) réaliser l'embarquement du navigateur autonome sur le satellite

\section{V de type faire $+\mathbf{N} \mathbf{2}+$ à bord de + dét $+\mathbf{N 1}$}

Ce type de fonctionnement n'est pas attesté dans le corpus étudié.

$$
\text { Vsupport de type faire + dét + DVB + de + N2 +à bord de + dét }+\mathbf{N}
$$

(16) faire le contrôle d' orbite à bord du satellite

(17) reléguer les prétraitements à bord du satellite

(18) réaliser les traitements à bord du satellite

(19) fournir des estimations de navigation à bord d' un satellite

(20) connaître les paramètres de navigation à bord du satellite

(21) réaliser l'application navigation à bord du satellite

(22) réaliser le contrôle d' orbite autonome à bord du satellite

(23) réaliser la probation en vol du calcul d'orbite à bord du satellite

\section{V de type transmettre + dét $+\mathbf{N} \mathbf{2}+\mathbf{a}+$ dét $+\mathbf{N 1}$}

(24) envoyer les paramètres d'orbite au satellite

\section{Vsupport de type faire + dét + DVB de type transmettre + de + N2 + à + dét + N1}

(25) réaliser l'envoi de télécommandes au satellite

(26) faire la transmission de téléchargements / télécommandes aux satellites

\section{V de mouvement + par/via + dét $+\mathbf{N 1}$}

(27) [les données de DIODE] descendent via le satellite

(28) [la voie de données] passe par le satellite

\subsubsection{Polyacception et point de vue}

Un expert du domaine a pu donner une identification à chacune de ces acceptions, identification que l'on peut considérer comme manifestant des points de vue, ou du moins les points de vue que cet expert perçoit au vu des résultats de l'analyse linguistique.

1. Le satellite peut être considéré comme un corps artificiel. Il s'agit, en somme, du sens du dictionnaire, qui présente le satellite (artificiel) comme un corps artificiel lancé de la terre de façon à devenir le satellite d'une planète.

2. Le satellite est considéré comme un mobile en ce sens qu'il constitue un corps qui peut être mû, dont on peut changer la position. Il s'agit donc d'une propriété du satellite qui est privilégiée dans ce type d'utilisation. 
3. La plupart des sens du terme satellite peuvent être interprétés comme une plateforme. On constate dans ce cas un glissement par métonymie puisque la plate-forme est une partie du satellite.

4. Par un glissement par métonymie, le satellite est considéré comme un véhicule.

5. Lorsqu'il est fait référence au satellite en tant qu'hôte, c'est une propriété (un trait dénotatif) du satellite qui est privilégiée.

6. Les fonctionnements dans lesquels le satellite est présenté comme un relais, une interface révèlent une autre propriété de l'objet satellite, en privilégiant également un trait dénotatif.

S'est posée alors la question de savoir si cette identification, a priori, de points de vue se retrouve dans l'analyse des résultats en fonction de l'origine des corpus étudiés. L'objectif était de vérifier si, pour chacune des deux séries de corpus (issus de deux divisions différentes) on pouvait identifier un patron syntaxico-sémantique dominant, c'est-à-dire une acception dominante.

Le tableau ci-dessous rend compte des résultats obtenus; chaque patron syntaxicosémantique a été étiqueté par le nom donné par l'expert. Chaque occurrence de satellite a été identifiée comme appartenant à l'une des classes de fonctionnement linguistique et comme provenant de l'une des deux divisions: D1 : «Observation de la terre», D2 : «Mathématiques Spatiales».

\begin{tabular}{|c|c|c|c|c|c|c|c|}
\hline & $\begin{array}{c}\text { corps } \\
\text { artificiel }\end{array}$ & mobile & plate-forme & véhicule & hôte & relais & \\
\hline D1 & $6.5 \%$ & $3.3 \%$ & 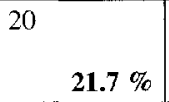 & - & - & - & $\begin{array}{l}29 \\
31.5 \%\end{array}$ \\
\hline $\mathrm{D} 2$ & $1.1 \%$ & $30.4 \%$ & $14.1 \%$ & $10.9 \%$ & $9.8 \%$ & $2.2 \%$ & $\begin{array}{l}63 \\
68.5 \%\end{array}$ \\
\hline & $7.6 \%$ & $33.7 \%$ & $35.8 \%$ & $10.9 \%$ & $9.8 \%$ & $2.2 \%$ & $\begin{array}{l}92 \\
100 \%\end{array}$ \\
\hline
\end{tabular}

Tableau 1:

Nombre d'apparitions (et pourcentages) des six points de vue sur le terme satellite dans le corpus

\subsubsection{Analyse des résultats}

L'analyse du tableau permet de tirer plusieurs conclusions.

1. Dans la division $D 1$, le point de vue plate-forme est nettement dominant $(21,7 \%$ des occurrences). De même, un type d'occurrences domine nettement dans la division $\mathrm{D} 2$, il s'agit des occurrences étiquetées mobile, avec 30,4\%.

Pour ce qui concerne ces classes d'acceptions (étiquetées mobile et plate-forme), on peut considérer qu'on a bien affaire à la manifestation d'un point de vue collectif. Ces deux classes d'acceptions sont dominantes dans la division «Observation de la terre» $(30,4 \%)$ et dans la division «Mathématiques Spatiales» $(21,7 \%)$ respectivement ce qui permet d'affirmer que c'est bien là le point de vue dominant d'une compétence socioprofessionnelle commune à un groupe de locuteurs identifié par la division à laquelle il appartient qui se manifeste. 
2. On peut remarquer que les acceptions dominantes, mobile $(33,7 \%)$ et plateforme $(35,8 \%)$ sont des acceptions attestées dans chacune des divisions, de même que la classe d'acceptions étiquetée corps artificiel qui est, quant à elle, très peu représentée. En faisant abstraction des points de vue dominants dans chaque division, on peut dire que les trois points de vue (mobile, plate-forme et corps artificiel) constituent les points de vue communs à l'ensemble des acteurs de l'entreprise CNES.

3. On peut noter que trois classes d'acceptions sont spécifiques à la division D2 (véhicule, hôte et relais) et qu'elles apparaissent avec une fréquence moins élevée que celle du point de vue dominant. On peut tirer deux conséquences de cet état de fait. D'une part, la présence d'un point de vue dominant (mobile) n'empêche pas la présence, dans le discours, de points de vue secondaires (véhicule, hôte, relais); d'autre part, il n'y a pas conflits de points de vue puisque tous les points de vue sont présents en D2. Les points de vue secondaires sont représentés par un nombre relativement faible d'occurrences (10 au plus); on pourrait alors considérer qu'il s'agit de points de vue individuels si l'on s'en tient au corpus étudié; il conviendrait néanmoins d'examiner d'autres textes afin de confirmer cette tendance.

\section{CONCLUSION}

Cet article nous a permis d'aborder la question de la polysémie dans les langues spécialisées à partir d'une synthèse des travaux sur la polysémie dans la langue générale. La notion du point de vue, associée en langue générale à celle de polysémie, nous a permis de faire un premier point sur des différences de fonctionnement en langue générale et en langue spécialisée. Nous avons considéré que les langues spécialisées avaient à la fois les caractéristiques d'un discours et celles d'une langue. Si l'on considère ce double fonctionnement, la notion de point de vue peut être associée à un choix individuel (le même que l'on trouve dans toute actualisation de langue en discours) ou à un choix collectif, celui qui fait qu'un ensemble de locuteurs découpent le monde de la même manière, celle qui correspond au domaine dans lequel ils évoluent. Dans une dernière partie, nous avons rendu compte d'une méthode de mise en évidence de cas de polyacceptions (manifestations linguistiques diverses d'un terme polysémique), et de l'existence de points de vue collectifs. L'existence de points de vue ne pose problème que s'ils n'ont pas été identifiés ou, plus rarement, que s'ils ne sont pas compatibles. Le rôle du linguiste-terminologue se borne à rendre compte de l'existence de points de vue différents.

Les résultats que nous présentons ici s'inscrivent dans une réflexion plus générale sur les rapports entre langue générale et langues spécialisées. Les besoins, les méthodes, la recherche même ayant évolué sur cette question, on ne peut plus se contenter de considérer que les langues spécialisées sont des sous-parties de la langue générale, donc sans grand intérêt spécifique. Chaque phénomène connu en langue doit être réexaminé dans les langues spécialisées afin de mettre au jour en quoi l'existence de contextes d'énonciation très spécifiques les modifient.

Notes

1. Notons qu'il n'en a pas toujours été ainsi et que, dans certains travaux, cette parenté est encore contesté au profit d'une perception logiciste de la terminologie ( $c f$. «La terminologie traduit dans ces préceptes les plus dogmatiques la survivance d'un positivisme révolu» (Slodzian 1994: 132)).

2. Alceste, Manuel du logiciel d'analyse de données textuelles, version 1.1, Société Image. 


\section{RÉFÉRENCES}

BAYLON, N. et M. FABRE (1978) : La sémantique, Paris, Nathan.

BEACCO, J.-C. et S. MOIRAND (1995): «Autour des discours de transmission de connaissances», Langue franģaise, 105, pp. 32-53.

CONDAMINES, A. (1993): «Un exemple d'utilisation de connaissances de sémantique lexicale : acquisition semi-automatique d'un vocabulaire de spécialité», Cahiers de lexicologie, 62 (1), pp. 25-65.

CONDAMINES, A. (1995) : «Terminology: New Needs, New Perspectives», Terminology, 2 (2), pp. $219-238$.

DESCAMPS, J.-L et coll. (1992) : Sémantique et concordances, coll. «Saint-Cloud», Paris, Klincksieck.

GENTILHOMME, Y. (1994): «Regards sur la terminologisation en lexicologie», Meta, 39 (4), pp. 546-560.

GUILBERT, L. (1973) «La spécificité du terme scientifique et technique», Langue française, 17, pp. 5-17.

KERBRAT-ORECCHIONI, C. (1977) : La connotation, Lyon, PUL.

KOCOUREK, R. (1991) : «Textes et termes», Meta, 36 (1), pp. 71-76.

LERAT, P. (1983) : Sémantique descriptive, Paris, PUF.

LERAT, P. (1995): Les langues spécialisées, Paris, PUF, 201 p.

LINTVELT, J. (1981) : Essai de typologie narrative: le «point de vue», Paris, Librairie Corti.

LYONS, J. (1990) : Sémantique linguistique, Larousse, Langue et Langage.

MARTIN, R. (1972): «Problèmes de sémantique lexicale», Travaux de linguistique et de littérature, 10 (1), pp. 11-124.

MARTINET, A. (1974) : «Homonymes et polysèmes», La linguistique, 10 (2), pp. 37-45.

MOESCHLER, et A. REBOUL (1994) : Dictionnaire encyclopédique de pragmatique, Paris, Seuil.

MORTUREUX, M.-F. (1995) : «Aspects linguistiques des vocabulaires scientifiques et techniques», Les Cannets du CEDISCOR, 3.

PICOCHE, J. (1984) : «Recherche de la cohérence des divers emplois du signe linguistique (fondamentale chez Guillaume) à travers la notion de signifié de puissance», Cahiers de lexicologie, 44 (1).

RASTIER, F., CAVAZZA, M. et A. ABEILLE (1995): Sémantique pour l'analyse: de la linguistique à l'informatique, Paris, Masson.

REBEYROLLE, J. (1995): Apports de la terminologie à l'étude des points de vue, Mémoire de fin de stage de DEA au CNES.

SAUSSURE, F. de (1983) : Cours de linguistique générale (1916), T. de Mauro (dir.), Paris, Payot.

SOLDZIAN, M. (1994) : «La doctrine terminologique, nouvelle théorie du signe au carrefour de l'universalisme et du logicisme», ALFA (Actes de Langue Française et de Linguistique) : Terminologie et langues de spécialité, $7 / 8$, pp. $121-136$. 